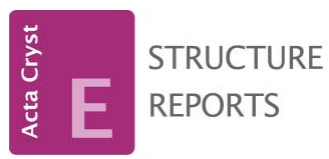

ISSN 1600-5368

Received 16 April 2014

Accepted 25 April 2014

Edited by W. T. A. Harrison, University of Aberdeen, Scotland

Keywords: crystal structure; lead; periodate; non-merohedral twinning

CCDC reference: 1004265

Supporting information: this article has supporting information at journals.iucr.org/e

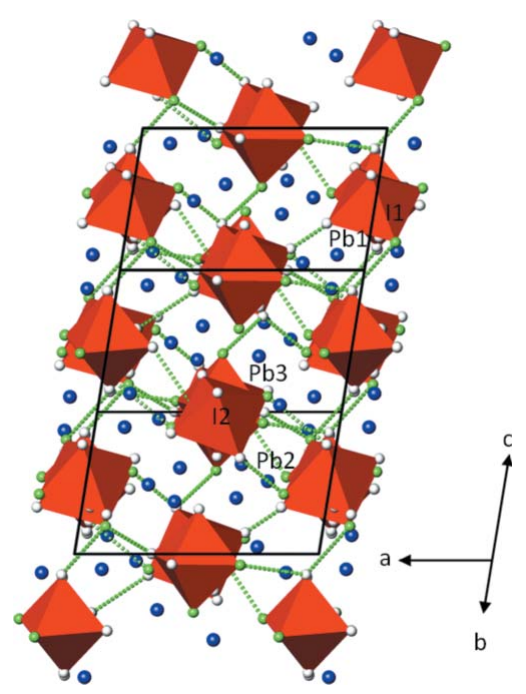

OPEN $\odot$ ACCESS

\section{Crystal structure of $\mathrm{Pb}_{3}\left(\mathrm{IO}_{4}(\mathrm{OH})_{2}\right)_{2}$}

\author{
Matthias Weil
}

Institute for Chemical Technologies and Analytics, Division of Structural Chemistry, Vienna University of Technology, Getreidemarkt 9/164-SC, A-1060 Vienna, Austria. *Correspondence e-mail: mweil@mail.zserv.tuwien.ac.at

The structure of the title compound, trilead(II) bis[dihydroxidotetraoxidoiodate(VII)], was determined from a crystal twinned by non-merohedry with two twin domains present [twin fraction 0.73 (1):0.27 (1)]. It contains three $\mathrm{Pb}^{2+}$ cations and two $\mathrm{IO}_{4}(\mathrm{OH})_{2}{ }^{3-}$ anions in the asymmetric unit. Each of the $\mathrm{Pb}^{2+}$ cations is surrounded by eight $\mathrm{O}$ atoms (cut-off value $=3.1 \AA$ ) in the form of a distorted polyhedron. The octahedral $\mathrm{IO}_{4}(\mathrm{OH})_{2}{ }^{3-}$ anions are arranged in rows extending parallel to [021], forming a distorted hexagonal rod packing. The cations and anions are linked into a framework structure. Although $\mathrm{H}$-atom positions could not be located, $\mathrm{O} \cdots \mathrm{O}$ distances suggest medium-strength hydrogen-bonding interactions between the $\mathrm{IO}_{4}(\mathrm{OH})_{2}$ octahedra, further consolidating the crystal packing.

\section{Chemical context}

Lead and mercury can both exist in different oxidation states and each of the two elements exhibits a peculiar crystal chemistry. In the case of $\mathrm{Pb}^{2+}$-containing compounds, the crystal chemistry is mainly dominated by the stereoactive $6 s^{2}$ lone-pair of lead (Holloway \& Melnik, 1997), whereas $\mathrm{Hg}^{2+}$-containing compounds show a strong preference for a linear coordination of mercury (Breitinger, 2004). In this respect, it appears surprising that for some $\mathrm{Pb}^{2+}$ - and $\mathrm{Hg}^{2+}$ containing compounds an isotypic relationship exists, e.g. for $\mathrm{PbAs}_{2} \mathrm{O}_{6}$ (Losilla et al., 1995) and $\mathrm{HgAs}_{2} \mathrm{O}_{6}$ (Mormann \& Jeitschko, 2000b; Weil, 2000), or for the mineral descloizite $\mathrm{PbZn}\left(\mathrm{VO}_{4}\right) \mathrm{OH}$ (Hawthorne \& Faggiani, 1979) and the synthetic phase $\mathrm{HgZn}\left(\mathrm{AsO}_{4}\right) \mathrm{OH}$ (Weil, 2004). With this in mind, it seemed interesting to study the relation between phases in the systems $\mathrm{Hg}^{\mathrm{II}}-\mathrm{I}^{\mathrm{VII}}-\mathrm{O}-\mathrm{H}$ and $\mathrm{Pb}^{\mathrm{II}}-\mathrm{I}^{\mathrm{VII}}-\mathrm{O}-\mathrm{H}$. Whereas in the system $\mathrm{Hg}^{\mathrm{II}}-\mathrm{I}^{\mathrm{VII}}-\mathrm{O}-\mathrm{H}$ two compounds have been structurally characterized, viz. $\mathrm{Hg}_{3}\left(\mathrm{IO}_{4}(\mathrm{OH})_{2}\right)_{2}$ (Mormann \& Jeitschko, 2000a) and $\mathrm{Hg}\left(\mathrm{IO}_{3}(\mathrm{OH})_{3}\right.$ ) (Mormann \& Jeitschko, 2001), a phase in the system $\mathrm{Pb}^{\mathrm{II}}-\mathrm{I}^{\mathrm{VII}}-\mathrm{O}-\mathrm{H}$ has not yet been structurally determined, although several lead(II) periodate phases have been reported to exist. Willard \& Thompson (1934) claimed to have obtained only one phase with composition $\mathrm{Pb}_{3} \mathrm{H}_{4}\left(\mathrm{IO}_{6}\right)_{2}$ in the system $\mathrm{Pb}^{\mathrm{II}}-\mathrm{I}^{\mathrm{VII}}-\mathrm{O}-\mathrm{H}$. However, Drátovský \& Matějčková $(1965 a, b)$ reported the existence of three phases with composition $\mathrm{Pb}_{3}\left(\mathrm{IO}_{5}\right)_{2} \cdot \mathrm{H}_{2} \mathrm{O}$, $\mathrm{Pb}_{2} \mathrm{I}_{2} \mathrm{O}_{9} \cdot 3 \mathrm{H}_{2} \mathrm{O}$ and $\mathrm{Pb}_{4} \mathrm{I}_{2} \mathrm{O}_{11} \cdot 5 \mathrm{H}_{2} \mathrm{O}$ in this system. To shed some light on the conflicting composition of the $\mathrm{Pb}: \mathrm{I} 3: 2$ phase $\left[\mathrm{Pb}_{3} \mathrm{H}_{4}\left(\mathrm{IO}_{6}\right)_{2}\right.$ versus $\mathrm{Pb}_{3}\left(\mathrm{IO}_{5}\right)_{2} \cdot \mathrm{H}_{2} \mathrm{O}$ with a lower water content], the synthetic procedure described by Willard \& Thompson (1934) was repeated for crystal growth of this lead periodate. The current structure determination of the obtained crystals showed the composition $\mathrm{Pb}_{3} \mathrm{H}_{4}\left(\mathrm{IO}_{6}\right)_{2}$ as reported by Willard \& Thompson (1934) to be correct. In a more reasonable crystal-chemical sense, the formula of these crystals should be rewritten as $\mathrm{Pb}_{3}\left(\mathrm{IO}_{4}(\mathrm{OH})_{2}\right)_{2}$. 


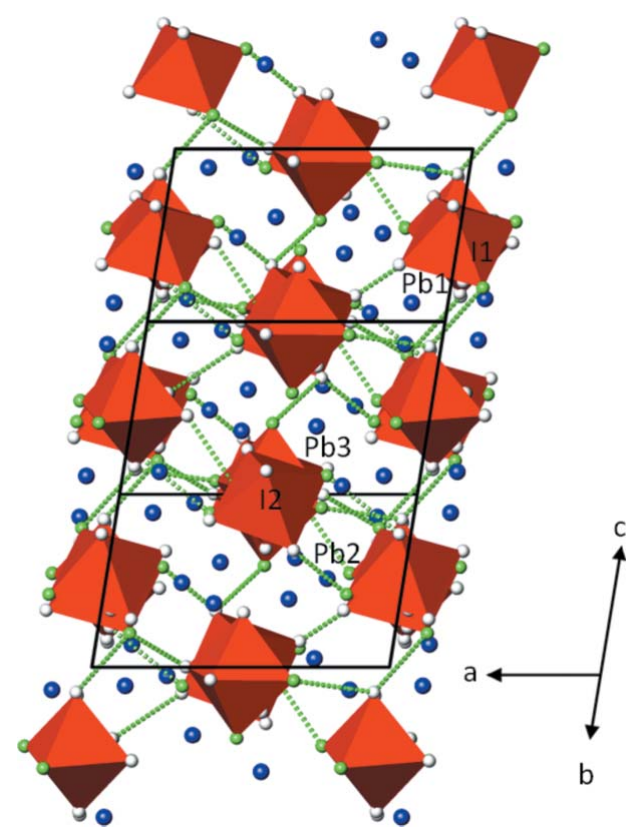

Figure 1

The crystal structure of $\mathrm{Pb}_{3}\left(\mathrm{IO}_{4}(\mathrm{OH})_{2}\right)_{2}$ in a projection along [021]. Displacement ellipsoids are drawn at the $90 \%$ probability level. O atoms bearing the $\mathrm{OH}$ function are given in green, the other $\mathrm{O}$ atoms are white. $\mathrm{Pb}-\mathrm{O}$ bonds are omitted for clarity; hydrogen-bonding interactions are shown as green dashed lines.

\section{Structural commentary}

Three $\mathrm{Pb}^{2+}$ cations and two $\mathrm{IO}_{4}(\mathrm{OH})_{2}{ }^{3-}$ octahedra are present in the asymmetric unit. The anions form a slightly distorted hexagonal rod packing with the rods extending parallel to [021]. Cations and anions are linked through common oxygen atoms into a framework structure (Fig. 1).

Each of the $\mathrm{Pb}^{2+}$ cations exhibits a coordination number of eight if $\mathrm{Pb}-\mathrm{O}$ interactions less than $3.1 \AA$ are considered to be relevant. The resulting $\left[\mathrm{PbO}_{8}\right]$ polyhedra are considerably distorted $[\mathrm{Pb}-\mathrm{O}$ distances range from $2.433(7)$ to 3.099 (8) $\AA$ ]. The stereochemical activity of the electron lone pairs in each of the polyhedra appears not to be very pronounced (Fig. 2).

Compounds and structures containing the periodate anion have been reviewed some time ago by Levason (1997). The compiled $\mathrm{I}-\mathrm{O}$ bond lengths are in good agreement with the two $\mathrm{IO}_{6}$ octahedra of the title compound, having a mean $\mathrm{I}-\mathrm{O}$ distance of $1.884 \AA$. Very similar mean values are found for comparable periodate compounds with large divalent cations, for example in $\mathrm{BaI}_{2} \mathrm{O}_{6}(\mathrm{OH})_{4} \cdot 2 \mathrm{H}_{2} \mathrm{O}$ (one $\mathrm{IO}_{6}$ octahedron, $1.895 \AA$; Häuseler, 2008), in $\mathrm{Ba}\left(\mathrm{IO}_{3}(\mathrm{OH})_{3}\right)$ (one $\mathrm{IO}_{6}$ octahedron, $1.879 \AA$ A; Sasaki et al., 1995), in $\mathrm{Hg}_{3}\left(\mathrm{IO}_{4}(\mathrm{OH})_{2}\right)_{2}$ (two $\mathrm{IO}_{6}$ octahedra, $1.888 \AA$; Mormann \& Jeitschko, 2000a) or in $\mathrm{Sr}\left(\mathrm{IO}_{2}(\mathrm{OH})_{4}\right)_{2} \cdot 3 \mathrm{H}_{2} \mathrm{O}$ (two $\mathrm{IO}_{6}$ octahedra, $1.888 \AA$; Alexandrova \& Häuseler, 2004).

Results of bond-valence calculations (Brown, 2002), using the parameters of Brese \& O'Keeffe (1991) for I-O bonds and of Krivovichev \& Brown (2001) for $\mathrm{Pb}-\mathrm{O}$ bonds, are reasonably close to the expected values (in valence units): $\mathrm{Pb} 1$ 1.89, Pb2 1.73, Pb3 1.89, I1 6.78, I2 6.90, O1 1.95, O2 1.49, O3
Table 1

Selected bond lengths $(\AA)$.

\begin{tabular}{|c|c|c|c|}
\hline $\mathrm{I} 1-\mathrm{O} 6$ & $1.845(8)$ & $\mathrm{I} 2-\mathrm{O} 11$ & $1.820(8)$ \\
\hline $\mathrm{I} 1-\mathrm{O} 3$ & $1.860(7)$ & $\mathrm{I} 2-\mathrm{O} 9$ & $1.850(8)$ \\
\hline $\mathrm{I} 1-\mathrm{O} 2^{\mathrm{i}}$ & $1.861(7)$ & $\mathrm{I} 2-\mathrm{O} 8$ & $1.855(7)$ \\
\hline $\mathrm{I} 1-\mathrm{O} 1^{\mathrm{ii}}$ & $1.877(7)$ & $\mathrm{I} 2-\mathrm{O} 7$ & $1.874(8)$ \\
\hline $\mathrm{I} 1-\mathrm{O} 5^{\mathrm{i}}$ & $1.920(8)$ & $\mathrm{I} 2-\mathrm{O} 12$ & $1.932(9)$ \\
\hline $\mathrm{I} 1-\mathrm{O} 4^{\mathrm{i}}$ & $1.956(8)$ & $\mathrm{I} 2-\mathrm{O} 10$ & $1.954(8)$ \\
\hline
\end{tabular}

Symmetry codes: (i) $-x, y-\frac{1}{2},-z+\frac{1}{2}$; (ii) $x,-y+\frac{1}{2}, z+\frac{1}{2}$.

Table 2

Hydrogen-bond geometry ( $\mathrm{\AA})$.

\begin{tabular}{|c|c|c|c|}
\hline$D-\mathrm{H} \cdots A$ & $D \cdots A$ & $D-\mathrm{H} \cdots A$ & $D \cdots A$ \\
\hline $\mathrm{O} 4 \cdots \mathrm{O} 7$ & 2.849 (11) & $\mathrm{O} 10 \cdots \mathrm{O} 11^{\text {iv }}$ & 2.675 (11) \\
\hline $\mathrm{O} 4 \cdots \mathrm{O} 2^{\mathrm{i}}$ & 2.849 (11) & $\mathrm{O} 12 \cdots \mathrm{O} 2^{\text {iv }}$ & $2.852(11)$ \\
\hline $\mathrm{O} 5 \cdots \mathrm{O} 11^{\mathrm{iii}}$ & $2.634(11)$ & & \\
\hline
\end{tabular}

1.90, O4 1.15, O5 1.15, O6 1.92, O7 1.98, O8 1.95, O 91.97 , O10 1.09, O11 1.34, O12 1.12. The $\mathrm{O}$ atoms involved in hydrogen bonding are readily identifiable. The donor $\mathrm{O}$ atoms $\mathrm{O} 4, \mathrm{O} 5$, $\mathrm{O} 10$ and $\mathrm{O} 12$ exhibit the longest $\mathrm{I}-\mathrm{O}$ bonds and the lowest bond-valence sums. Atom $\mathrm{O} 11$ has also a low bond-valence sum, explainable by its role as a twofold acceptor atom of medium-strength hydrogen-bonding interactions (Table 2) that additionally stabilize the packing of the structure (Fig. 1).

Comparison of the structures of $\mathrm{Pb}_{3}\left(\mathrm{IO}_{4}(\mathrm{OH})_{2}\right)_{2}$ and of $\mathrm{Hg}_{3}\left(\mathrm{IO}_{4}(\mathrm{OH})_{2}\right)_{2} \quad\left[P 2_{1} / c ; \quad Z=4, a=8.5429(7), \quad b=\right.$ 12.2051 (8) $\AA, c=9.3549$ (8) $\left.\AA, \beta=90.884(7)^{\circ}\right]$ reveals some close similarities. A 'true' isotypic relationship (Lima-de-Faria et al., 1990) is difficult to derive for the two structures.
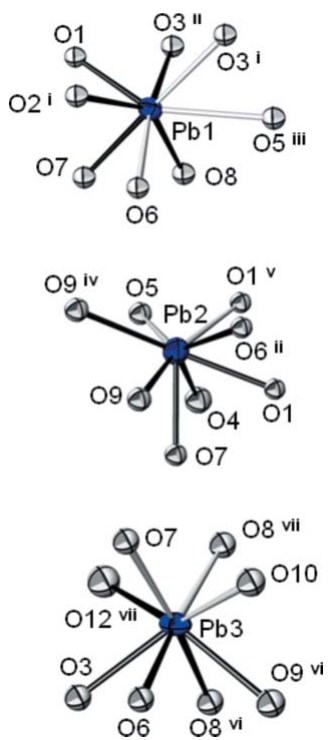

Figure 2

Coordination polyhedra of the three $\mathrm{Pb}^{2+}$ cations in the structure of $\mathrm{Pb}_{3}\left(\mathrm{IO}_{4}(\mathrm{OH})_{2}\right)_{2}$. Bonds shorter than $2.7 \AA$ are given by solid black lines, longer bonds between 2.7 and $3.1 \AA$ as open black lines. Displacement ellipsoids are drawn at the $90 \%$ probability level. [Symmetry codes: (i) $-x, y-\frac{1}{2},-z+\frac{1}{2}$; (ii) $x,-y+\frac{1}{2}, z-\frac{1}{2}$; (iii) $x, y-1, z$; (iv) $-x+1,-y+1$, $-z$; (v) $-x,-y+1,-z$; (vi) $x,-y+\frac{1}{2}, z+\frac{1}{2}$; (vii) $-x+1, y+\frac{1}{2},-z+\frac{1}{2}$; (viii) $-x, y+\frac{1}{2},-z+\frac{1}{2}$.] 


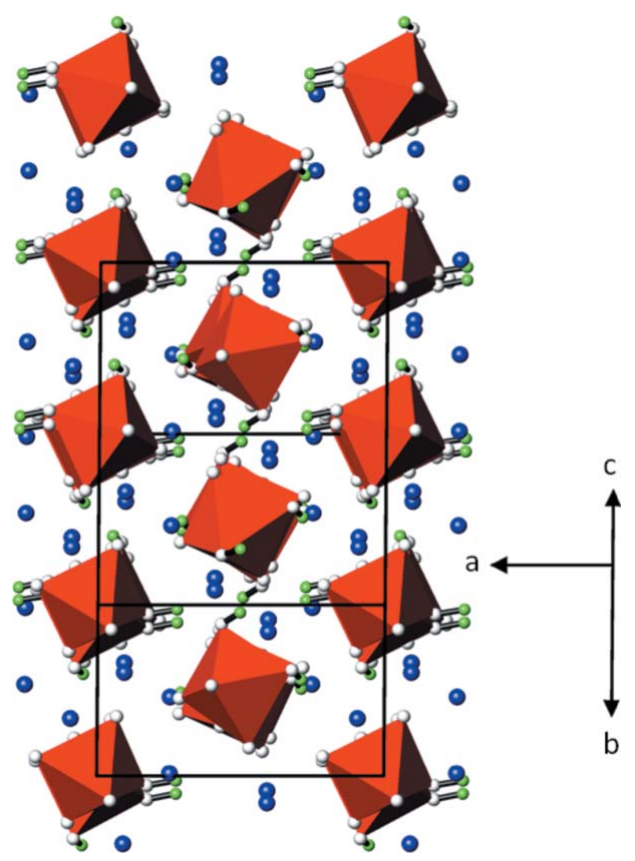

Figure 3

The crystal structure of $\mathrm{Hg}_{3}\left(\mathrm{IO}_{4}(\mathrm{OH})_{2}\right)_{2}$ (Mormann \& Jeitschko, 2000a) in a projection along [011]. Colour code as in Fig. $1 . \mathrm{Hg}-\mathrm{O}$ and $\mathrm{O}-$ $\mathrm{H} \cdots \mathrm{O}$ interactions are omitted for clarity.

However, they are isopointal and show the same type of arrangement in terms of the crystal packing. In the mercury compound, the $\mathrm{IO}_{4}(\mathrm{OH})_{2}{ }^{3-}$ octahedra are likewise hexagonally packed in rods (Fig. 3). The cations are situated in between this arrangement which is further consolidated by $\mathrm{O}-\mathrm{H} \cdots \mathrm{O}$ hydrogen bonding.

\section{Synthesis and crystallization}

The preparation conditions described by Willard \& Thompson (1934) were modified slightly. Instead of using $\mathrm{NaIO}_{4}$ as the periodate source, periodic acid was employed.

$1.25 \mathrm{~g} \mathrm{~Pb}\left(\mathrm{NO}_{3}\right)_{2}$ was dissolved in $25 \mathrm{ml}$ water, acidified with a few drops of concentrated nitric acid and heated until boiling. Then the periodic acid solution $(0.85 \mathrm{~g}$ in $25 \mathrm{ml}$ water $)$ was slowly added to the lead solution. The addition of the first portion of the periodic acid solution ( $c a .3-4 \mathrm{ml})$ resulted in an off-white precipitate near the drop point that redissolved under stirring. After further addition, the precipitate remained and changed the colour in the still boiling solution from off-white to yellow-orange within half an hour. After filtration of the precipitate, a few colourless crystals of the title compound formed in the mother liquor on cooling. X-ray powder diffraction data of the polycrystalline precipitate are in very good agreement with simulated data based on the refinement of $\mathrm{Pb}_{3}\left(\mathrm{IO}_{4}(\mathrm{OH})_{2}\right)_{2}$.

\section{Refinement}

All investigated crystals were twinned by non-merohedry. Intensity data of the measured crystal could be indexed to belong to two domains, with a refined twin domain ratio of
Table 3

Experimental details.

\begin{tabular}{|c|c|}
\hline \multicolumn{2}{|l|}{ Crystal data } \\
\hline Chemical formula & $\mathrm{Pb}_{3}\left[\mathrm{IO}_{4}(\mathrm{OH})_{2}\right]_{2}$ \\
\hline$M_{\mathrm{r}}$ & 1071.40 \\
\hline Crystal system, space group & Monoclinic, $P 2_{1} / c$ \\
\hline Temperature $(\mathrm{K})$ & 296 \\
\hline$a, b, c(\AA)$ & $8.9653(9), 9.2113(9), 12.8052(13)$ \\
\hline$\beta\left({ }^{\circ}\right)$ & $101.042(2)$ \\
\hline$V\left(\mathrm{~A}^{3}\right)$ & $1037.90(18)$ \\
\hline$Z$ & 4 \\
\hline Radiation type & Мо $K \alpha$ \\
\hline$\mu\left(\mathrm{mm}^{-1}\right)$ & 54.55 \\
\hline Crystal size $(\mathrm{mm})$ & $0.06 \times 0.06 \times 0.05$ \\
\hline \multicolumn{2}{|l|}{ Data collection } \\
\hline Diffractometer & Siemens SMART CCD \\
\hline Absorption correction & $\begin{array}{l}\text { Multi-scan (TWINABS; Bruker, } \\
\text { 2008) }\end{array}$ \\
\hline$T_{\min }, T_{\max }$ & $0.253,0.746$ \\
\hline $\begin{array}{l}\text { No. of measured, independent and } \\
\text { observed }[I>2 \sigma(I)] \text { reflections }\end{array}$ & $3196,3196,2587$ \\
\hline$(\sin \theta / \lambda)_{\max }\left(\AA^{-1}\right)$ & 0.716 \\
\hline \multicolumn{2}{|l|}{ Refinement } \\
\hline$R\left[F^{2}>2 \sigma\left(F^{2}\right)\right], w R\left(F^{2}\right), S$ & $0.041,0.087,1.07$ \\
\hline No. of reflections & 3196 \\
\hline No. of parameters & 94 \\
\hline $\mathrm{H}$-atom treatment & $\mathrm{H}$-atom parameters not refined \\
\hline$\Delta \rho_{\max }, \Delta \rho_{\min }\left(\mathrm{e} \AA^{-3}\right)$ & $2.88,-1.95$ \\
\hline
\end{tabular}

Computer programs: SMART (Bruker, 2008), SAINT-Plus (Bruker, 2008), SHELXS97 and SHELXL97 (Sheldrick, 2008), ATOMS for Windows (Dowty, 2006) and publCIF (Westrip, 2010).

0.73 (1):0.27 (1). Reflections originating from the minor component as well as overlapping reflections of the two domains (less than $10 \%$ of all measured reflections) were separated and excluded. The $\mathrm{H}$ atoms of the $\mathrm{IO}_{4}(\mathrm{OH})_{2}$ octahedra could not be located from difference maps and were therefore not considered in the final model. The $\mathrm{O}$ atoms were refined with isotropic displacement parameters. The remaining maximum and minimum electron densities are found 0.73 and $0.68 \AA$, respectively, from atom $\mathrm{Pb} 2$. Structure data were finally standardized with STRUCTURE-TIDY (Gelato \& Parthé, 1987). It should be noted that the intensity statistics point to a pronounced $C$-centred basis cell (space group $C 2 / c$ with lattice parameters of $a \simeq 14.16, b \simeq 9.21, c \simeq$ $8.97 \AA, \beta \simeq 117.4^{\circ}$ ) with weak superstructure reflections violating the $C$-centering.

\section{Acknowledgements}

The X-ray centre of the Vienna University of Technology is acknowledged for providing access to the single-crystal diffractometer.

\section{References}

Alexandrova, M. \& Häuseler, H. (2004). J. Mol. Struct. 706, 7-13. Breitinger, D. K. (2004). Cadmium and Mercury, in Comprehensive Coordination Chemistry II, edited by J. A. McCleverty \& T. J. Meyer, ch. 6, pp. 1253-1292. Oxford: Elsevier.

Brese, N. E. \& O'Keeffe, M. (1991). Acta Cryst. B47, 192-197.

Brown, I. D. (2002). In The Chemical Bond in Inorganic Chemistry: The Bond Valence Model. Oxford University Press. 
Bruker (2008). SMART, SAINT-Plus and TWINABS. Bruker AXS Inc., Madison, Wisconsin, USA.

Dowty, E. (2006). ATOMS for Windows. Shape Software, Kingsport, Tennessee, USA.

Drátovský, M. \& Matějčková, J. (1965a). Chem. Zvesti, 19, 604-610.

Drátovský, M. \& Matějčková, J. (1965b). Chem. Zvesti, 19, 447-455.

Gelato, L. M. \& Parthé, E. (1987). J. Appl. Cryst. 20, 139-143.

Häuseler, H. (2008). J. Mol. Struct. 892, 1-7.

Hawthorne, F. C. \& Faggiani, R. (1979). Acta Cryst. B35, 717-720.

Holloway, C. E. \& Melnik, M. (1997). Main Group Met. Chem. 20, 583-625.

Krivovichev, S. V. \& Brown, I. D. (2001). Z. Kristallogr. 216, 245-247. Levason, W. (1997). Coord. Chem. Rev. 161, 33-79.

Lima-de-Faria, J., Hellner, E., Liebau, F., Makovicky, E. \& Parthé, E. (1990). Acta Cryst. A46, 1-11.
Losilla, E. R., Aranda, M. A. G., Ramirez, F. J. \& Bruque, S. (1995). J. Phys. Chem. 99, 12975-12979.

Mormann, T. J. \& Jeitschko, W. (2000a). Z. Kristallogr. New Cryst. Struct., 215, 315-316.

Mormann, T. J. \& Jeitschko, W. (2000b). Z. Kristallogr. New Cryst. Struct., 215, 471-472.

Mormann, T. J. \& Jeitschko, W. (2001). Z. Kristallogr. New Cryst. Struct., 216, 1-2.

Sasaki, M., Yarita, T. \& Sato, S. (1995). Acta Cryst. C51, 1968-1970.

Sheldrick, G. M. (2008). Acta Cryst. A64, 112-122.

Weil, M. (2000). Z. Naturforsch. Teil B, 55, 699-706.

Weil, M. (2004). Acta Cryst. E60, i25-i27.

Westrip, S. P. (2010). J. Appl. Cryst. 43, 920-925.

Willard, H. H. \& Thompson, J. J. (1934). J. Am. Chem. Soc. 56, 18281830. 


\section{supporting information}

Acta Cryst. (2014). E70, 14-17 [https://doi.org/10.1107/S1600536814009520]

\section{Crystal structure of $\mathrm{Pb}_{3}\left(\mathrm{IO}_{4}(\mathrm{OH})_{2}\right)_{2}$}

\section{Matthias Weil}

\section{Computing details}

Data collection: SMART (Bruker, 2008); cell refinement: SAINT-Plus (Bruker, 2008); data reduction: SAINT-Plus (Bruker, 2008); program(s) used to solve structure: SHELXS97 (Sheldrick, 2008); program(s) used to refine structure: SHELXL97 (Sheldrick, 2008); molecular graphics: ATOMS for Windows (Dowty, 2006); software used to prepare material for publication: publCIF (Westrip, 2010).

Trilead(II) bis[dihydroxidotetraoxidoiodate(VII)]

Crystal data

$\mathrm{Pb}_{3}\left[\mathrm{IO}_{4}(\mathrm{OH})_{2}\right]_{2}$

$M_{r}=1071.40$

Monoclinic, $P 2_{1} / c$

Hall symbol: -P 2ybc

$a=8.9653(9) \AA$

$b=9.2113(9) \AA$

$c=12.8052(13) \AA$

$\beta=101.042(2)^{\circ}$

$V=1037.90(18) \AA^{3}$

$Z=4$

\section{Data collection}

Siemens SMART CCD diffractometer

Radiation source: fine-focus sealed tube Graphite monochromator $\omega$ scans

Absorption correction: multi-scan

(TWINABS; Bruker, 2008)

$T_{\min }=0.253, T_{\max }=0.746$

\section{Refinement}

Refinement on $F^{2}$

Least-squares matrix: full

$R\left[F^{2}>2 \sigma\left(F^{2}\right)\right]=0.041$

$w R\left(F^{2}\right)=0.087$

$S=1.07$

3196 reflections

94 parameters

0 restraints
$F(000)=1808$

$D_{\mathrm{x}}=6.857 \mathrm{Mg} \mathrm{m}^{-3}$

Mo $K \alpha$ radiation, $\lambda=0.71073 \AA$

Cell parameters from 3673 reflections

$\theta=3.2-30.5^{\circ}$

$\mu=54.55 \mathrm{~mm}^{-1}$

$T=296 \mathrm{~K}$

Block, colourless

$0.06 \times 0.06 \times 0.05 \mathrm{~mm}$

3196 measured reflections

3196 independent reflections

2587 reflections with $I>2 \sigma(I)$

$R_{\text {int }}=0.000$

$\theta_{\text {max }}=30.6^{\circ}, \theta_{\min }=2.3^{\circ}$

$h=-12 \rightarrow 12$

$k=0 \rightarrow 13$

$l=0 \rightarrow 18$

Primary atom site location: structure-invariant direct methods

Secondary atom site location: difference Fourier map

$\mathrm{H}$-atom parameters not refined

$w=1 /\left[\sigma^{2}\left(F_{\mathrm{o}}^{2}\right)+(0.0319 P)^{2}+17.8096 P\right]$

where $P=\left(F_{\mathrm{o}}{ }^{2}+2 F_{\mathrm{c}}{ }^{2}\right) / 3$

$(\Delta / \sigma)_{\max }<0.001$

$\Delta \rho_{\max }=2.88 \mathrm{e} \AA^{-3}$

$\Delta \rho_{\min }=-1.95$ e $\AA^{-3}$ 


\section{Special details}

Geometry. All e.s.d.'s (except the e.s.d. in the dihedral angle between two 1.s. planes) are estimated using the full covariance matrix. The cell e.s.d.'s are taken into account individually in the estimation of e.s.d.'s in distances, angles and torsion angles; correlations between e.s.d.'s in cell parameters are only used when they are defined by crystal symmetry. An approximate (isotropic) treatment of cell e.s.d.'s is used for estimating e.s.d.'s involving 1.s. planes.

Refinement. Refinement of $F^{2}$ against ALL reflections. The weighted $R$-factor $w R$ and goodness of fit $S$ are based on $F^{2}$, conventional $R$-factors $R$ are based on $F$, with $F$ set to zero for negative $F^{2}$. The threshold expression of $F^{2}>\sigma\left(F^{2}\right)$ is used only for calculating $R$-factors (gt) etc. and is not relevant to the choice of reflections for refinement. $R$-factors based on $F^{2}$ are statistically about twice as large as those based on $F$, and $R$ - factors based on ALL data will be even larger.

Fractional atomic coordinates and isotropic or equivalent isotropic displacement parameters $\left(\hat{A}^{2}\right)$

\begin{tabular}{lllll}
\hline & $x$ & $y$ & $z$ & $U_{\text {iso }} * / U_{\text {eq }}$ \\
\hline $\mathrm{Pb} 1$ & $0.12192(5)$ & $0.13042(4)$ & $0.11904(3)$ & $0.01318(10)$ \\
$\mathrm{Pb} 2$ & $0.25685(5)$ & $0.51903(5)$ & $0.01540(4)$ & $0.01842(11)$ \\
$\mathrm{Pb3}$ & $0.37507(5)$ & $0.36874(5)$ & $0.38134(3)$ & $0.01409(10)$ \\
$\mathrm{I} 1$ & $0.00670(7)$ & $0.23313(7)$ & $0.36046(5)$ & $0.00838(13)$ \\
$\mathrm{I} 2$ & $0.50186(7)$ & $0.24738(6)$ & $0.14267(5)$ & $0.00735(13)$ \\
O1 & $0.0343(9)$ & $0.3332(8)$ & $0.0015(6)$ & $0.0110(14)^{*}$ \\
O2 & $0.0389(9)$ & $0.7922(8)$ & $0.2811(6)$ & $0.0125(15)^{*}$ \\
O3 & $0.1058(9)$ & $0.4046(8)$ & $0.4090(6)$ & $0.0122(15)^{*}$ \\
O4 & $0.1088(10)$ & $0.5549(9)$ & $0.1797(6)$ & $0.0178(17)^{*}$ \\
O5 & $0.1831(9)$ & $0.8100(8)$ & $0.1159(6)$ & $0.0129(15)^{*}$ \\
O6 & $0.1842(9)$ & $0.1429(8)$ & $0.3433(6)$ & $0.0117(15)^{*}$ \\
O7 & $0.3161(9)$ & $0.3260(8)$ & $0.1618(6)$ & $0.0121(15)^{*}$ \\
O8 & $0.4052(9)$ & $0.0832(8)$ & $0.0785(6)$ & $0.0121(15)^{*}$ \\
O9 & $0.4802(9)$ & $0.3391(8)$ & $0.0121(6)$ & $0.0144(16)^{*}$ \\
O10 & $0.5247(9)$ & $0.1474(8)$ & $0.2794(6)$ & $0.0145(16)^{*}$ \\
O11 & $0.6146(10)$ & $0.3912(8)$ & $0.2170(6)$ & $0.0158(16)^{*}$ \\
O12 & $0.6856(10)$ & $0.1562(9)$ & $0.1172(6)$ & $0.0182(17)^{*}$ \\
& & & & \\
\hline
\end{tabular}

Atomic displacement parameters $\left(\AA^{2}\right)$

\begin{tabular}{lllllll}
\hline & $U^{11}$ & $U^{22}$ & $U^{33}$ & $U^{12}$ & $U^{13}$ & $U^{23}$ \\
\hline $\mathrm{Pb} 1$ & $0.0134(2)$ & $0.01402(19)$ & $0.01215(19)$ & $-0.00236(15)$ & $0.00243(15)$ & $0.00042(14)$ \\
$\mathrm{Pb} 2$ & $0.0168(2)$ & $0.01424(19)$ & $0.0236(2)$ & $0.00084(17)$ & $0.00251(16)$ & $0.00040(16)$ \\
$\mathrm{Pb} 3$ & $0.0153(2)$ & $0.0173(2)$ & $0.01036(18)$ & $-0.00366(16)$ & $0.00426(15)$ & $-0.00050(14)$ \\
$\mathrm{I} 1$ & $0.0075(3)$ & $0.0104(3)$ & $0.0072(3)$ & $-0.0003(2)$ & $0.0014(2)$ & $0.0006(2)$ \\
$\mathrm{I} 2$ & $0.0066(3)$ & $0.0078(3)$ & $0.0076(3)$ & $-0.0002(2)$ & $0.0011(2)$ & $-0.0005(2)$ \\
\hline
\end{tabular}

Geometric parameters $\left(A,{ }^{\circ}\right)$

\begin{tabular}{llll}
\hline $\mathrm{Pb} 1-\mathrm{O} 1$ & $2.433(7)$ & $\mathrm{Pb} 3-\mathrm{O} 9^{\text {vi }}$ & $2.599(8)$ \\
$\mathrm{Pb} 1-\mathrm{O} 7$ & $2.493(8)$ & $\mathrm{Pb} 3-\mathrm{O} 6$ & $2.678(8)$ \\
$\mathrm{Pb} 1-\mathrm{O} 2^{\mathrm{i}}$ & $2.577(8)$ & $\mathrm{Pb} 3-\mathrm{O} 12^{\text {vii }}$ & $2.704(8)$ \\
$\mathrm{Pb} 1-\mathrm{O} 3^{\text {ii }}$ & $2.685(7)$ & $\mathrm{Pb} 3-\mathrm{O} 8^{\text {vi }}$ & $2.767(8)$ \\
$\mathrm{Pb} 1-\mathrm{O} 8$ & $2.723(8)$ & $\mathrm{Pb} 3-\mathrm{O} 7$ & $2.787(7)$ \\
$\mathrm{Pb} 1-\mathrm{O} 6$ & $2.821(7)$ & $\mathrm{Pb} 3-\mathrm{O} 10$ & $2.886(8)$ \\
$\mathrm{Pb} 1-\mathrm{O} 3^{\mathrm{i}}$ & $2.888(8)$ & $\mathrm{I} 1-\mathrm{O} 6$ & $1.845(8)$
\end{tabular}




\begin{tabular}{|c|c|c|c|}
\hline $\mathrm{Pb} 1-\mathrm{O}^{\mathrm{iii}}$ & $3.004(7)$ & $\mathrm{I} 1-\mathrm{O} 3$ & $1.860(7)$ \\
\hline $\mathrm{Pb} 2-\mathrm{O} 7$ & $2.564(7)$ & $\mathrm{I} 1-\mathrm{O} 2^{\mathrm{i}}$ & $1.861(7)$ \\
\hline $\mathrm{Pb} 2-\mathrm{O} 9$ & $2.606(8)$ & $\mathrm{I} 1-\mathrm{O} 1^{\mathrm{vi}}$ & $1.877(7)$ \\
\hline $\mathrm{Pb} 2-\mathrm{O} 1$ & $2.609(7)$ & $\mathrm{I} 1-\mathrm{O} 5^{\mathrm{i}}$ & $1.920(8)$ \\
\hline $\mathrm{Pb} 2-\mathrm{O}^{\mathrm{ii}}$ & $2.638(7)$ & $\mathrm{I} 1-\mathrm{O} 4^{\mathrm{i}}$ & $1.956(8)$ \\
\hline $\mathrm{Pb} 2-\mathrm{O} 4$ & $2.714(8)$ & $\mathrm{I} 2-\mathrm{O} 11$ & $1.820(8)$ \\
\hline $\mathrm{Pb} 2-\mathrm{O}^{\mathrm{iv}}$ & $2.777(8)$ & $\mathrm{I} 2-\mathrm{O} 9$ & $1.850(8)$ \\
\hline $\mathrm{Pb} 2-\mathrm{O} 1^{\mathrm{v}}$ & $2.915(7)$ & $\mathrm{I} 2-\mathrm{O} 8$ & $1.855(7)$ \\
\hline $\mathrm{Pb} 2-\mathrm{O} 5$ & $3.099(8)$ & $\mathrm{I} 2-\mathrm{O} 7$ & $1.874(8)$ \\
\hline $\mathrm{Pb} 3-\mathrm{O}^{\mathrm{vi}}$ & $2.527(7)$ & $\mathrm{I} 2-\mathrm{O} 12$ & $1.932(9)$ \\
\hline $\mathrm{Pb} 3-\mathrm{O} 3$ & $2.528(8)$ & $\mathrm{I} 2-\mathrm{O} 10$ & $1.954(8)$ \\
\hline $\mathrm{O} 1-\mathrm{Pb} 1-\mathrm{O} 7$ & $73.1(2)$ & $\mathrm{O} 7-\mathrm{I} 2-\mathrm{O} 10$ & $90.5(3)$ \\
\hline $\mathrm{O} 1-\mathrm{Pb} 1-\mathrm{O} 2^{\mathrm{i}}$ & $73.6(2)$ & $\mathrm{O} 12-\mathrm{I} 2-\mathrm{O} 10$ & $90.0(3)$ \\
\hline $\mathrm{O} 7-\mathrm{Pb} 1-\mathrm{O} 2^{\mathrm{i}}$ & $84.6(2)$ & 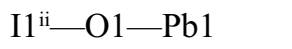 & $108.2(3)$ \\
\hline $\mathrm{O} 1-\mathrm{Pb} 1-\mathrm{O}^{\mathrm{ii}}$ & $61.5(2)$ & $\mathrm{I} 1^{\mathrm{ii}}-\mathrm{O} 1-\mathrm{Pb} 2$ & $103.7(3)$ \\
\hline $\mathrm{O} 7-\mathrm{Pb} 1-\mathrm{O}^{3 i}$ & $102.0(2)$ & $\mathrm{Pb} 1-\mathrm{O} 1-\mathrm{Pb} 2$ & $108.0(3)$ \\
\hline $\mathrm{O} 2^{\mathrm{i}}-\mathrm{Pb} 1-\mathrm{O}^{\mathrm{ii}}$ & $129.7(2)$ & 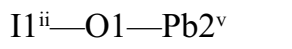 & $97.4(3)$ \\
\hline $\mathrm{O} 1-\mathrm{Pb} 1-\mathrm{O} 8$ & $102.0(2)$ & $\mathrm{Pb} 1-\mathrm{O} 1-\mathrm{Pb} 2^{\mathrm{v}}$ & $125.6(3)$ \\
\hline $\mathrm{O} 7-\mathrm{Pb} 1-\mathrm{O} 8$ & $61.3(2)$ & $\mathrm{Pb} 2-\mathrm{O} 1-\mathrm{Pb} 2^{\mathrm{v}}$ & $111.1(2)$ \\
\hline $\mathrm{O} 2^{\mathrm{i}}-\mathrm{Pb} 1-\mathrm{O} 8$ & $144.9(2)$ & $\mathrm{I} 1^{\mathrm{ii}-} \mathrm{O} 1-\mathrm{Pb} 3^{\mathrm{ii}}$ & $57.6(2)$ \\
\hline $\mathrm{O} 3^{\mathrm{ii}}-\mathrm{Pb} 1-\mathrm{O} 8$ & $70.3(2)$ & $\mathrm{Pb} 1-\mathrm{O} 1-\mathrm{Pb} 3^{\mathrm{ii}}$ & $73.14(18)$ \\
\hline $\mathrm{O} 1-\mathrm{Pb} 1-\mathrm{O} 6$ & $125.2(2)$ & $\mathrm{Pb} 2-\mathrm{O} 1-\mathrm{Pb} 3^{\mathrm{ii}}$ & $73.13(17)$ \\
\hline $\mathrm{O} 7-\mathrm{Pb} 1-\mathrm{O} 6$ & $75.7(2)$ & $\mathrm{Pb} 2^{\mathrm{v}}-\mathrm{O} 1-\mathrm{Pb} 3^{\mathrm{ii}}$ & $154.3(2)$ \\
\hline $\mathrm{O} 2 \mathrm{i}-\mathrm{Pb} 1-\mathrm{O} 6$ & $59.4(2)$ & 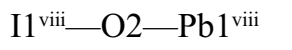 & $106.0(3)$ \\
\hline $\mathrm{O} 3^{\mathrm{ii}}-\mathrm{Pb} 1-\mathrm{O} 6$ & $170.7(2)$ & $\mathrm{I} 1^{\mathrm{viii}}-\mathrm{O} 2-\mathrm{Pb} 2^{\mathrm{ix}}$ & $156.0(4)$ \\
\hline $\mathrm{O} 8-\mathrm{Pb} 1-\mathrm{O} 6$ & $101.0(2)$ & $\mathrm{Pb} 1^{\mathrm{vii}}-\mathrm{O} 2-\mathrm{Pb} 2^{\mathrm{ix}}$ & $97.4(2)$ \\
\hline $\mathrm{O} 1-\mathrm{Pb} 1-\mathrm{O} 3^{\mathrm{i}}$ & $109.8(2)$ & $\mathrm{I}{ }^{\mathrm{viii}}-\mathrm{O} 2-\mathrm{Pb} 1^{\mathrm{x}}$ & $75.1(2)$ \\
\hline $\mathrm{O} 7-\mathrm{Pb} 1-\mathrm{O}^{\mathrm{i}}$ & $174.4(2)$ & $\mathrm{Pb} 1^{\mathrm{vii}}-\mathrm{O} 2-\mathrm{Pb}^{\mathrm{x}}$ & $155.4(3)$ \\
\hline $\mathrm{O} 2^{\mathrm{i}}-\mathrm{Pb} 1-\mathrm{O} 3^{\mathrm{i}}$ & $91.7(2)$ & $\mathrm{Pb} 2^{\mathrm{ix}}-\mathrm{O} 2-\mathrm{Pb} 1^{\mathrm{x}}$ & $86.03(16)$ \\
\hline $\mathrm{O} 3^{\mathrm{ii}}-\mathrm{Pb} 1-\mathrm{O} 3^{\mathrm{i}}$ & $83.5(2)$ & $\mathrm{I} 1^{\mathrm{viii}}-\mathrm{O} 2-\mathrm{Pb} 3^{\text {viii }}$ & $62.0(2)$ \\
\hline $\mathrm{O} 8-\mathrm{Pb} 1-\mathrm{O}^{\mathrm{i}}$ & $121.6(2)$ & $\mathrm{Pb} 1^{\text {viii }}-\mathrm{O} 2-\mathrm{Pb} 3^{\text {viii }}$ & $78.83(19)$ \\
\hline $\mathrm{O} 6-\mathrm{Pb} 1-\mathrm{O} 3^{\mathrm{i}}$ & $98.8(2)$ & $\mathrm{Pb} 2^{\mathrm{ix}}-\mathrm{O} 2-\mathrm{Pb} 3^{\text {viii }}$ & $129.9(2)$ \\
\hline $\mathrm{O} 1-\mathrm{Pb} 1-\mathrm{O} 5^{\mathrm{iii}}$ & $141.7(2)$ & $\mathrm{Pb} 1^{\mathrm{x}}-\mathrm{O} 2-\mathrm{Pb} 3^{\text {viii }}$ & $80.39(14)$ \\
\hline $\mathrm{O} 7-\mathrm{Pb} 1-\mathrm{O} 5^{\mathrm{iii}}$ & $126.3(2)$ & $\mathrm{I} 1-\mathrm{O} 3-\mathrm{Pb} 3$ & $104.5(3)$ \\
\hline $\mathrm{O} 2^{\mathrm{i}}-\mathrm{Pb} 1-\mathrm{O} 5^{\mathrm{iii}}$ & $134.1(2)$ & $\mathrm{I} 1-\mathrm{O} 3-\mathrm{Pb} 1^{\mathrm{vi}}$ & $99.4(3)$ \\
\hline $\mathrm{O} 3^{\mathrm{ii}}-\mathrm{Pb} 1-\mathrm{O}^{\mathrm{iii}}$ & $81.0(2)$ & $\mathrm{Pb} 3-\mathrm{O} 3-\mathrm{Pb} 1^{\mathrm{vi}}$ & $104.8(3)$ \\
\hline $\mathrm{O} 8-\mathrm{Pb} 1-\mathrm{O} 5^{\mathrm{iii}}$ & $70.2(2)$ & $\mathrm{I} 1-\mathrm{O} 3-\mathrm{Pb} 1^{\text {viii }}$ & $106.8(3)$ \\
\hline $\mathrm{O} 6-\mathrm{Pb} 1-\mathrm{O} 5^{\mathrm{iii}}$ & $93.0(2)$ & $\mathrm{Pb} 3-\mathrm{O} 3-\mathrm{Pb} 1^{\text {viii }}$ & $138.4(3)$ \\
\hline $\mathrm{O} 3^{\mathrm{i}}-\mathrm{Pb} 1-\mathrm{O} 5^{\mathrm{iii}}$ & $54.4(2)$ & 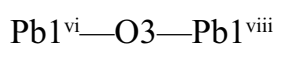 & $96.5(2)$ \\
\hline $\mathrm{O} 7-\mathrm{Pb} 2-\mathrm{O} 9$ & $61.2(2)$ & $\mathrm{I}{ }^{\mathrm{vii}}-\mathrm{O} 4-\mathrm{Pb} 2$ & $102.2(3)$ \\
\hline $\mathrm{O} 7-\mathrm{Pb} 2-\mathrm{O} 1$ & $69.1(2)$ & $\mathrm{I} 1^{\mathrm{vii}}-\mathrm{O} 4-\mathrm{Pb} 3$ & $146.5(3)$ \\
\hline $\mathrm{O} 9-\mathrm{Pb} 2-\mathrm{O} 1$ & $99.3(2)$ & $\mathrm{Pb} 2-\mathrm{O} 4-\mathrm{Pb} 3$ & $98.2(2)$ \\
\hline $\mathrm{O} 7-\mathrm{Pb} 2-\mathrm{O}^{\mathrm{ii}}$ & $101.7(2)$ & $\mathrm{I} 1^{\mathrm{viii}}-\mathrm{O} 4-\mathrm{Pb} 1^{\mathrm{viii}}$ & $71.6(2)$ \\
\hline $\mathrm{O} 9-\mathrm{Pb} 2-\mathrm{O}^{\mathrm{ii}}$ & $72.2(2)$ & $\mathrm{Pb} 2-\mathrm{O} 4-\mathrm{Pb} 1^{\text {viii }}$ & $173.4(3)$ \\
\hline $\mathrm{O} 1-\mathrm{Pb} 2-\mathrm{O}^{\mathrm{ii}}$ & $60.6(2)$ & $\mathrm{Pb} 3-\mathrm{O} 4-\mathrm{Pb} 1^{\text {viii }}$ & $88.39(17)$ \\
\hline $\mathrm{O} 7-\mathrm{Pb} 2-\mathrm{O} 4$ & $65.3(2)$ & $\mathrm{I} 1^{\mathrm{viii}}-\mathrm{O} 4-\mathrm{Pb} 2^{\mathrm{v}}$ & $68.3(2)$ \\
\hline $\mathrm{O} 9-\mathrm{Pb} 2-\mathrm{O} 4$ & $125.5(2)$ & $\mathrm{Pb} 2-\mathrm{O} 4-\mathrm{Pb} 2^{\mathrm{v}}$ & $87.5(2)$ \\
\hline
\end{tabular}




\begin{tabular}{|c|c|c|c|}
\hline $\mathrm{O} 1-\mathrm{Pb} 2-\mathrm{O} 4$ & $69.6(2)$ & $\mathrm{Pb} 3-\mathrm{O} 4-\mathrm{Pb} 2^{\mathrm{v}}$ & $139.5(2)$ \\
\hline $\mathrm{O}^{\mathrm{ii}}-\mathrm{Pb} 2-\mathrm{O} 4$ & $129.6(2)$ & $\mathrm{Pb}^{\mathrm{viii}}-\mathrm{O} 4-\mathrm{Pb} 2^{\mathrm{v}}$ & $87.94(18)$ \\
\hline $\mathrm{O} 7-\mathrm{Pb} 2-\mathrm{O}^{\mathrm{iv}}$ & $110.9(2)$ & $\mathrm{I} 1^{\mathrm{viii}-\mathrm{O} 4-\mathrm{Pb} 1}$ & $143.6(3)$ \\
\hline $\mathrm{O} 9-\mathrm{Pb} 2-\mathrm{O} 9^{\mathrm{iv}}$ & $67.9(3)$ & $\mathrm{Pb} 2-\mathrm{O} 4-\mathrm{Pb} 1$ & $72.12(18)$ \\
\hline $\mathrm{O} 1-\mathrm{Pb} 2-\mathrm{O}^{\mathrm{iv}}$ & $163.1(2)$ & $\mathrm{Pb} 3-\mathrm{O} 4-\mathrm{Pb} 1$ & $68.44(14)$ \\
\hline $\mathrm{O}^{\mathrm{ii}}-\mathrm{Pb} 2-\mathrm{O}^{\mathrm{iv}}$ & $103.9(2)$ & 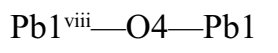 & $111.3(2)$ \\
\hline $\mathrm{O} 4-\mathrm{Pb} 2-\mathrm{O}^{\mathrm{iv}}$ & $126.5(2)$ & $\mathrm{Pb} 2^{\mathrm{v}}-\mathrm{O} 4-\mathrm{Pb} 1$ & $75.48(15)$ \\
\hline $\mathrm{O} 7-\mathrm{Pb} 2-\mathrm{O}^{\mathrm{v}}$ & $115.8(2)$ & $\mathrm{I} 1^{\mathrm{viii}-\mathrm{O} 5-\mathrm{Pb} 1^{\mathrm{x}}}$ & $101.0(3)$ \\
\hline $\mathrm{O} 9-\mathrm{Pb} 2-\mathrm{O}^{\mathrm{v}}$ & $167.4(2)$ & $\mathrm{I} 1^{\mathrm{viii}-\mathrm{O} 5-\mathrm{Pb} 2}$ & $90.7(3)$ \\
\hline $\mathrm{O} 1-\mathrm{Pb} 2-\mathrm{O} 1^{\mathrm{v}}$ & $68.9(2)$ & $\mathrm{Pb}^{\mathrm{x}}-\mathrm{O} 5-\mathrm{Pb} 2$ & $155.4(3)$ \\
\hline $\mathrm{O} 6^{\mathrm{ii}}-\mathrm{Pb} 2-\mathrm{O} 1^{\mathrm{v}}$ & $97.3(2)$ & 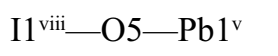 & $69.1(2)$ \\
\hline $\mathrm{O} 4-\mathrm{Pb} 2-\mathrm{O}^{\mathrm{v}}$ & $55.7(2)$ & $\mathrm{Pb}^{\mathrm{x}}-\mathrm{O} 5-\mathrm{Pb}^{\mathrm{v}}$ & $75.90(16)$ \\
\hline $\mathrm{O} 9^{\mathrm{iv}}-\mathrm{Pb} 2-\mathrm{O}^{\mathrm{v}}$ & $122.7(2)$ & $\mathrm{Pb} 2-\mathrm{O} 5-\mathrm{Pb}^{\mathrm{v}}$ & $88.49(18)$ \\
\hline $\mathrm{O} 7-\mathrm{Pb} 2-\mathrm{O} 5$ & $109.1(2)$ & $\mathrm{I}^{\mathrm{viii}}-\mathrm{O} 5-\mathrm{Pb} 3^{\mathrm{vii}}$ & $163.1(3)$ \\
\hline $\mathrm{O} 9-\mathrm{Pb} 2-\mathrm{O} 5$ & $141.5(2)$ & $\mathrm{Pb} 1^{x}-\mathrm{O} 5-\mathrm{Pb} 3^{\mathrm{vii}}$ & $92.88(19)$ \\
\hline $\mathrm{O} 1-\mathrm{Pb} 2-\mathrm{O} 5$ & $112.0(2)$ & $\mathrm{Pb} 2-\mathrm{O} 5-\mathrm{Pb} 3^{\text {vii }}$ & $80.25(17)$ \\
\hline $\mathrm{O} 6{ }^{\mathrm{ii}}-\mathrm{Pb} 2-\mathrm{O} 5$ & $142.9(2)$ & $\mathrm{Pb} 1^{\mathrm{v}}-\mathrm{O} 5-\mathrm{Pb} 3^{\mathrm{vii}}$ & $124.4(2)$ \\
\hline $\mathrm{O} 4-\mathrm{Pb} 2-\mathrm{O} 5$ & $53.0(2)$ & $\mathrm{I} 1-\mathrm{O} 6-\mathrm{Pb} 2^{\mathrm{vi}}$ & $103.6(3)$ \\
\hline $\mathrm{O} 9^{\mathrm{iv}}-\mathrm{Pb} 2-\mathrm{O} 5$ & $84.2(2)$ & $\mathrm{I} 1-\mathrm{O} 6-\mathrm{Pb} 3$ & $99.4(3)$ \\
\hline $\mathrm{O} 1^{\mathrm{v}}-\mathrm{Pb} 2-\mathrm{O} 5$ & $50.8(2)$ & $\mathrm{Pb} 2^{\mathrm{vi}}-\mathrm{O} 6-\mathrm{Pb} 3$ & $103.9(3)$ \\
\hline $\mathrm{O}^{\mathrm{vi}}-\mathrm{Pb} 3-\mathrm{O} 3$ & $76.1(3)$ & $\mathrm{I} 1-\mathrm{O} 6-\mathrm{Pb} 1$ & $97.6(3)$ \\
\hline $\mathrm{O} 8^{\mathrm{vi}}-\mathrm{Pb} 3-\mathrm{O}^{\mathrm{vi}}$ & $61.9(2)$ & $\mathrm{Pb} 2^{\mathrm{vi}}-\mathrm{O} 6-\mathrm{Pb} 1$ & $142.8(3)$ \\
\hline $\mathrm{O} 3-\mathrm{Pb} 3-\mathrm{O} 9^{\mathrm{vi}}$ & $104.1(2)$ & $\mathrm{Pb} 3-\mathrm{O} 6-\mathrm{Pb} 1$ & $102.2(2)$ \\
\hline $\mathrm{O} 88^{\mathrm{vi}}-\mathrm{Pb} 3-\mathrm{O} 6$ & $105.1(2)$ & $\mathrm{I} 2-\mathrm{O} 7-\mathrm{Pb} 1$ & $107.0(3)$ \\
\hline $\mathrm{O} 3-\mathrm{Pb} 3-\mathrm{O} 6$ & $62.2(2)$ & $\mathrm{I} 2-\mathrm{O} 7-\mathrm{Pb} 2$ & $103.7(3)$ \\
\hline $\mathrm{O} 9^{\mathrm{vi}}-\mathrm{Pb} 3-\mathrm{O} 6$ & $71.7(2)$ & $\mathrm{Pb} 1-\mathrm{O} 7-\mathrm{Pb} 2$ & $107.6(3)$ \\
\hline $\mathrm{O} 8^{\mathrm{vi}}-\mathrm{Pb} 3-\mathrm{O} 12^{\mathrm{vii}}$ & $78.7(2)$ & $\mathrm{I} 2-\mathrm{O} 7-\mathrm{Pb} 3$ & $100.6(3)$ \\
\hline $\mathrm{O} 3-\mathrm{Pb} 3-\mathrm{O} 12^{\mathrm{vii}}$ & $70.9(3)$ & $\mathrm{Pb} 1-\mathrm{O} 7-\mathrm{Pb} 3$ & $108.2(3)$ \\
\hline $\mathrm{O} 9^{\mathrm{vi}}-\mathrm{Pb} 3-\mathrm{O} 12^{\mathrm{vii}}$ & $140.0(2)$ & $\mathrm{Pb} 2-\mathrm{O} 7-\mathrm{Pb} 3$ & $127.7(3)$ \\
\hline $\mathrm{O} 6-\mathrm{Pb} 3-\mathrm{O} 12^{\mathrm{vii}}$ & $129.8(2)$ & $\mathrm{I} 2-\mathrm{O} 7-\mathrm{Pb} 3^{\mathrm{ii}}$ & $56.96(19)$ \\
\hline $\mathrm{O} 8^{\mathrm{vi}}-\mathrm{Pb} 3-\mathrm{O} 8^{\mathrm{vii}}$ & $75.7(3)$ & $\mathrm{Pb} 1-\mathrm{O} 7-\mathrm{Pb} 3^{\mathrm{ii}}$ & $72.30(17)$ \\
\hline $\mathrm{O} 3-\mathrm{Pb} 3-\mathrm{O} 8^{\mathrm{vii}}$ & $123.1(2)$ & $\mathrm{Pb} 2-\mathrm{O} 7-\mathrm{Pb} 3^{\mathrm{ii}}$ & $73.10(17)$ \\
\hline $\mathrm{O} 9^{\mathrm{vi}}-\mathrm{Pb} 3-\mathrm{O} 8^{\mathrm{vii}}$ & $104.4(2)$ & $\mathrm{Pb} 3-\mathrm{O} 7-\mathrm{Pb} 3^{\mathrm{ii}}$ & $154.9(3)$ \\
\hline $\mathrm{O} 6-\mathrm{Pb} 3-\mathrm{O} 8^{\mathrm{vii}}$ & $174.5(2)$ & $\mathrm{I} 2-\mathrm{O} 8-\mathrm{Pb} 3^{\mathrm{ii}}$ & $104.5(3)$ \\
\hline $\mathrm{O} 12^{\mathrm{vii}-\mathrm{Pb} 3-\mathrm{O}^{\mathrm{vii}}}$ & $55.7(2)$ & $\mathrm{I} 2-\mathrm{O} 8-\mathrm{Pb} 1$ & $99.1(3)$ \\
\hline $\mathrm{O} 8^{\mathrm{vi}-\mathrm{Pb} 3-\mathrm{O} 7}$ & $174.9(2)$ & $\mathrm{Pb} 3{ }^{\mathrm{ii}}-\mathrm{O} 8-\mathrm{Pb} 1$ & $103.7(3)$ \\
\hline $\mathrm{O} 3-\mathrm{Pb} 3-\mathrm{O} 7$ & $99.1(2)$ & $\mathrm{I} 2-\mathrm{O} 8-\mathrm{Pb}^{\mathrm{xi}}$ & $104.1(3)$ \\
\hline $\mathrm{O} 9^{\mathrm{vi}}-\mathrm{Pb} 3-\mathrm{O} 7$ & $121.4(2)$ & $\mathrm{Pb} 3^{\mathrm{ii}}-\mathrm{O} 8-\mathrm{Pb}^{\mathrm{xi}}$ & $104.3(3)$ \\
\hline $\mathrm{O} 6-\mathrm{Pb} 3-\mathrm{O} 7$ & $73.5(2)$ & $\mathrm{Pb} 1-\mathrm{O} 8-\mathrm{Pb} 3^{\mathrm{xi}}$ & $137.3(3)$ \\
\hline $\mathrm{O} 12^{\mathrm{vii}-\mathrm{Pb} 3-\mathrm{O} 7}$ & $98.4(2)$ & $\mathrm{I} 2-\mathrm{O} 9-\mathrm{Pb} 3^{\mathrm{ii}}$ & $102.0(3)$ \\
\hline 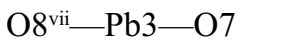 & $106.2(2)$ & $\mathrm{I} 2-\mathrm{O} 9-\mathrm{Pb} 2$ & $102.9(3)$ \\
\hline $\mathrm{O} 8^{\mathrm{vi}}-\mathrm{Pb} 3-\mathrm{O} 10$ & $127.3(2)$ & $\mathrm{Pb} 3{ }^{\mathrm{ii}}-\mathrm{O} 9-\mathrm{Pb} 2$ & $107.1(3)$ \\
\hline $\mathrm{O} 3-\mathrm{Pb} 3-\mathrm{O} 10$ & $134.0(2)$ & $\mathrm{I} 2-\mathrm{O} 9-\mathrm{Pb} 2^{\mathrm{iv}}$ & $112.6(4)$ \\
\hline 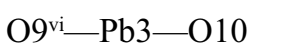 & $68.2(2)$ & $\mathrm{Pb}^{3}{ }^{\mathrm{ii}}-\mathrm{O} 9-\mathrm{Pb} 2^{\mathrm{iv}}$ & $118.5(3)$ \\
\hline $\mathrm{O} 6-\mathrm{Pb} 3-\mathrm{O} 10$ & $72.9(2)$ & $\mathrm{Pb} 2-\mathrm{O} 9-\mathrm{Pb} 2^{\mathrm{iv}}$ & $112.1(3)$ \\
\hline $\mathrm{O} 12^{\mathrm{vii}-\mathrm{Pb} 3-\mathrm{O} 10}$ & $143.8(2)$ & $\mathrm{I} 2-\mathrm{O} 10-\mathrm{Pb} 3$ & $95.4(3)$ \\
\hline $\mathrm{O} 8^{\mathrm{vii}}-\mathrm{Pb} 3-\mathrm{O} 10$ & $102.3(2)$ & $\mathrm{I} 2-\mathrm{O} 10-\mathrm{Pb} 2^{\mathrm{xi}}$ & $148.8(4)$ \\
\hline $\mathrm{O} 7-\mathrm{Pb} 3-\mathrm{O} 10$ & $57.2(2)$ & $\mathrm{Pb} 3-\mathrm{O} 10-\mathrm{Pb}^{\mathrm{xi}}$ & $98.9(2)$ \\
\hline
\end{tabular}




\begin{tabular}{|c|c|c|c|}
\hline $\mathrm{O} 6-\mathrm{I} 1-\mathrm{O} 3$ & $93.1(3)$ & $\mathrm{I} 2-\mathrm{O} 10-\mathrm{Pb}^{\mathrm{xi}}$ & $79.3(2)$ \\
\hline $\mathrm{O} 6-\mathrm{I} 1-\mathrm{O} 2^{\mathrm{i}}$ & $92.8(3)$ & $\mathrm{Pb} 3-\mathrm{O} 10-\mathrm{Pb} 3^{\mathrm{xi}}$ & $167.0(3)$ \\
\hline $\mathrm{O} 3-\mathrm{I} 1-\mathrm{O} 2^{\mathrm{i}}$ & $94.6(3)$ & $\mathrm{Pb} 2^{\mathrm{xi}}-\mathrm{O} 10-\mathrm{Pb} 3^{\mathrm{xi}}$ & $91.50(19)$ \\
\hline $\mathrm{O} 6-\mathrm{I} 1-\mathrm{O}^{\mathrm{vi}}$ & $90.6(3)$ & $\mathrm{I} 2-\mathrm{O} 10-\mathrm{Pb} 1$ & $67.0(2)$ \\
\hline $\mathrm{O} 3-\mathrm{I} 1-\mathrm{O} 1^{\mathrm{vi}}$ & $89.3(3)$ & $\mathrm{Pb} 3-\mathrm{O} 10-\mathrm{Pb} 1$ & $78.32(18)$ \\
\hline $\mathrm{O} 2^{\mathrm{i}}-\mathrm{I} 1-\mathrm{O} 1^{\mathrm{vi}}$ & $174.7(3)$ & $\mathrm{Pb} 2^{\mathrm{xi}}-\mathrm{O} 10-\mathrm{Pb} 1$ & $143.2(2)$ \\
\hline $\mathrm{O} 6-\mathrm{I} 1-\mathrm{O} 5^{\mathrm{i}}$ & $174.5(3)$ & $\mathrm{Pb}^{3 \mathrm{xi}}-\mathrm{O} 10-\mathrm{Pb} 1$ & $88.65(17)$ \\
\hline $\mathrm{O} 3-\mathrm{I} 1-\mathrm{O} 5^{\mathrm{i}}$ & $90.9(3)$ & $\mathrm{I} 2-\mathrm{O} 11-\mathrm{Pb} 3$ & $85.6(3)$ \\
\hline $\mathrm{O} 2^{\mathrm{i}}-\mathrm{I} 1-\mathrm{O} 5^{\mathrm{i}}$ & $90.5(3)$ & $\mathrm{I} 2-\mathrm{O} 11-\mathrm{Pb} 2^{\mathrm{iv}}$ & $88.1(3)$ \\
\hline $\mathrm{O} 1^{\mathrm{vi}}-\mathrm{I} 1-\mathrm{O} 5^{\mathrm{i}}$ & $85.8(3)$ & $\mathrm{Pb} 3-\mathrm{O} 11-\mathrm{Pb} 2^{\mathrm{iv}}$ & $157.4(3)$ \\
\hline $\mathrm{O} 6-\mathrm{I} 1-\mathrm{O} 4^{\mathrm{i}}$ & $90.9(3)$ & $\mathrm{I} 2-\mathrm{O} 11-\mathrm{Pb} 1^{\mathrm{vii}}$ & $171.0(4)$ \\
\hline $\mathrm{O} 3-\mathrm{I} 1-\mathrm{O} 4^{\mathrm{i}}$ & $174.5(3)$ & $\mathrm{Pb} 3-\mathrm{O} 11-\mathrm{Pb} 1^{\mathrm{vii}}$ & $95.8(2)$ \\
\hline $\mathrm{O} 2^{\mathrm{i}}-\mathrm{I} 1-\mathrm{O} 4^{\mathrm{i}}$ & $89.0(3)$ & $\mathrm{Pb} 2^{\mathrm{iv}}-\mathrm{O} 11-\mathrm{Pb} 1^{\mathrm{vii}}$ & $93.74(19)$ \\
\hline $\mathrm{O} 1^{\mathrm{vi}}-\mathrm{I} 1-\mathrm{O} 4^{\mathrm{i}}$ & $86.9(3)$ & $\mathrm{I} 2-\mathrm{O} 11-\mathrm{Pb} 2$ & $64.5(2)$ \\
\hline 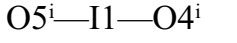 & $84.8(3)$ & $\mathrm{Pb} 3-\mathrm{O} 11-\mathrm{Pb} 2$ & $83.53(18)$ \\
\hline $\mathrm{O} 11-\mathrm{I} 2-\mathrm{O} 9$ & $95.3(3)$ & $\mathrm{Pb}^{2 \mathrm{iv}}-\mathrm{O} 11-\mathrm{Pb} 2$ & $74.20(15)$ \\
\hline $\mathrm{O} 11-\mathrm{I} 2-\mathrm{O} 8$ & $172.0(3)$ & $\mathrm{Pb} 1^{\mathrm{vii}}-\mathrm{O} 11-\mathrm{Pb} 2$ & $124.5(2)$ \\
\hline $\mathrm{O} 9-\mathrm{I} 2-\mathrm{O} 8$ & $90.7(3)$ & $\mathrm{I} 2-\mathrm{O} 12-\mathrm{Pb}^{\mathrm{xi}}$ & $104.2(4)$ \\
\hline $\mathrm{O} 11-\mathrm{I} 2-\mathrm{O} 7$ & 93.9 (4) & $\mathrm{I} 2-\mathrm{O} 12-\mathrm{Pb} 2^{\mathrm{iv}}$ & $85.5(3)$ \\
\hline $\mathrm{O} 9-\mathrm{I} 2-\mathrm{O} 7$ & $90.0(3)$ & $\mathrm{Pb} 3^{\mathrm{xi}}-\mathrm{O} 12-\mathrm{Pb} 2^{\mathrm{iv}}$ & $151.9(3)$ \\
\hline $\mathrm{O} 8-\mathrm{I} 2-\mathrm{O} 7$ & $91.2(3)$ & $\mathrm{I} 2-\mathrm{O} 12-\mathrm{Pb} 3^{\mathrm{ii}}$ & $68.4(2)$ \\
\hline $\mathrm{O} 11-\mathrm{I} 2-\mathrm{O} 12$ & $89.9(4)$ & $\mathrm{Pb}^{\mathrm{xi}}-\mathrm{O} 12-\mathrm{Pb}^{\mathrm{ii}}$ & $79.9(2)$ \\
\hline $\mathrm{O} 9-\mathrm{I} 2-\mathrm{O} 12$ & $89.5(4)$ & $\mathrm{Pb} 2^{\mathrm{iv}}-\mathrm{O} 12-\mathrm{Pb} 3^{3 i}$ & $79.42(16)$ \\
\hline $\mathrm{O} 8-\mathrm{I} 2-\mathrm{O} 12$ & $84.9(3)$ & $\mathrm{I} 2-\mathrm{O} 12-\mathrm{Pb}^{\mathrm{xii}}$ & $155.4(4)$ \\
\hline $\mathrm{O} 7-\mathrm{I} 2-\mathrm{O} 12$ & $176.1(3)$ & $\mathrm{Pb} 3^{\mathrm{xi}}-\mathrm{O} 12-\mathrm{Pb} 1^{\mathrm{xii}}$ & $98.2(2)$ \\
\hline $\mathrm{O} 11-\mathrm{I} 2-\mathrm{O} 10$ & $85.5(3)$ & $\mathrm{Pb} 2^{\mathrm{iv}}-\mathrm{O} 12-\mathrm{Pb} 1^{\mathrm{xii}}$ & $79.41(17)$ \\
\hline $\mathrm{O} 9-\mathrm{I} 2-\mathrm{O} 10$ & $179.1(3)$ & $\mathrm{Pb} 3^{\mathrm{ii}}-\mathrm{O} 12-\mathrm{Pb} 1^{\mathrm{xii}}$ & $126.6(2)$ \\
\hline $\mathrm{O} 8-\mathrm{I} 2-\mathrm{O} 10$ & $88.4(3)$ & & \\
\hline
\end{tabular}

Symmetry codes: (i) $-x, y-1 / 2,-z+1 / 2$; (ii) $x,-y+1 / 2, z-1 / 2$; (iii) $x, y-1, z$; (iv) $-x+1,-y+1,-z$; (v) $-x,-y+1,-z$; (vi) $x,-y+1 / 2, z+1 / 2$; (vii) $-x+1, y+1 / 2$, $-z+1 / 2$; (viii) $-x, y+1 / 2,-z+1 / 2$; (ix) $x,-y+3 / 2, z+1 / 2$; (x) $x, y+1, z$; (xi) $-x+1, y-1 / 2,-z+1 / 2$; (xii) $x+1, y, z$.

Hydrogen-bond geometry $(\AA)$

\begin{tabular}{ll}
\hline$D-\mathrm{H} \cdots A$ & $D \cdots A$ \\
\hline $\mathrm{O} 4 \cdots \mathrm{O} 7$ & $2.849(11)$ \\
$\mathrm{O} 4 \cdots \mathrm{O} 2^{\mathrm{i}}$ & $2.849(11)$ \\
$\mathrm{O} 5 \cdots \mathrm{O} 11^{\mathrm{vii}}$ & $2.634(11)$ \\
$\mathrm{O} 10 \cdots \mathrm{O} 11^{\mathrm{xi}}$ & $2.675(11)$ \\
$\mathrm{O} 12 \cdots \mathrm{O} 2^{\mathrm{xi}}$ & $2.852(11)$ \\
\hline
\end{tabular}

Symmetry codes: (i) $-x, y-1 / 2,-z+1 / 2$; (vii) $-x+1, y+1 / 2,-z+1 / 2 ;$ (xi) $-x+1, y-1 / 2,-z+1 / 2$. 